\title{
Wave Analysis of a Diffusive Modified Leslie-Gower Predator-prey System with Holling Type IV Schemes
}

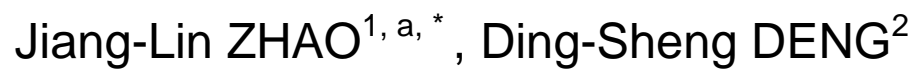 \\ ${ }^{1}$ Department of Mathematics, Sichuan Minzu College, Kangding, 626001, China \\ ${ }^{2}$ Department of Computer Science, Sichuan Minzu College, Kangding, 626001, China \\ aemail: ws05101162@163.com \\ ${ }^{*}$ Corresponding author
}

Keywords: Leslie-Gower, Holling type IV schemes, Traveling wave solution.

\begin{abstract}
A diffusive predator-prey model with modified Leslie-Gower and Holling type IV schemes is investigated analytically and numerically. Mathematical theoretical works mainly focus on the existence of traveling wave solutions. Numerical simulations are performed to confirm the feasibility of traveling wave solutions. All these results are significant in exploration of the dynamic complexity of ecosystems.
\end{abstract}

\section{Introduction}

Predator-prey interactions have attracted considerable attention in mathematical biology. In the classical Lotka-Volterra predator-prey models, the growth rate of population is assumed to be proportional to its size. However, Leslie and Gower [1,2] initiated a predator-prey model where the carrying capacity of predator is proportional to the number of prey. Recently, the non-monotonic Holling type IV functional response has been widely used to describe the process of predation with self-selection and the inhibitory effect of prey $[3,4,5]$. On the other hand, all organisms interact with each other in a limited space. Meanwhile, the spatial value has been regarded as a pivotal role on how ecological communities are shaped $[6,7,8]$. From above, we can study the following equations:

$$
\left\{\begin{array}{l}
\frac{\partial H}{\partial T}=d_{1} \Delta H+H\left(a_{1}-b_{1} H\right)-\frac{c_{1} H P}{e_{1}+H^{2}}, \\
\frac{d P}{d T}=d_{2} \Delta P+P\left(a_{2}-\frac{c_{2} P}{e_{2}+H}\right),
\end{array}\right.
$$

where $H(T, X)$ and $P(T, X)$ denote the densities of prey and predator at time $T$ and position $X, a_{1}$ and $a_{2}$ correspond to the growth rates of prey and predator, $b_{1}$ reflects the competitive strength among individuals of prey, $c_{1}$ is the maximum value of the per capita reduction of $H$ due to $P, c_{2} / a_{2}$ measures the ration of prey to support one predator, $e_{1}$ is interpreted as the half-saturation constant, $e_{2}$ indicates the quality of the alternative that provides the environment, $\Delta$ is the Laplacian operator, $d_{1}$ and $d_{2}$ are the diffusion coefficients of prey and predator. All the parameters of Eq. 1 are supposed to be positive. For simplicity, the scaling transformation is performed: $t=a_{1} T$, 
$x=\left(\frac{a_{1}}{d_{1}}\right)^{\frac{1}{2}}, u(t, x)=\frac{b_{1}}{a_{1}} H(T, X), \quad w(t, x)=\frac{b_{1} c_{2}}{a_{1} a_{2}} P(T, X), \quad s=\frac{a_{2}}{a_{1}}, \quad \delta=\frac{d_{2}}{d_{1}}$, $a=\frac{e_{1} b_{1}^{2}}{a_{1}^{2}}, b=\frac{b_{1} e_{2}}{a_{1}}, \alpha=\frac{c_{1} c_{2} b_{1}^{3}}{a_{2} a_{1}^{3}}$. Assume that the interaction between prey and predator is limited by the bounded region $\Omega$ in $R^{n}$ with the smooth boundary. This leads to the following spatial-temporal equations:

$$
\left\{\begin{array}{l}
\frac{\partial u}{\partial t}=\Delta u+u(1-u)-\frac{\alpha u w}{a+u^{2}}, x \in \Omega, t>0 \\
\frac{\partial w}{\partial t}=\delta \Delta w+s w\left(1-\frac{w}{b+u}\right), x \in \Omega, t>0 \\
\frac{\partial u}{\partial n}=\frac{\partial w}{\partial n}=0, \quad x \in \partial \Omega, t>0, \\
u(x, 0)=u_{0}(x), w(x, 0)=w_{0}(x), x \in \Omega,
\end{array}\right.
$$

where $\partial / \partial n$ is differential in the direction of the outward unit normal to $\partial \Omega$. Clearly, Eq. 2 has the following equilibrium points:

$E_{0}=(0,0), E_{1}=(1,0), E_{2}=(0, b), E_{3}=\left(u_{*}, w_{*}\right)=\left(b+u_{*}, w_{*}\right)$,

where $w_{*}$ is the positive root of $u^{3}-u^{2}+(a+\alpha) u+\alpha b-a=0$.

\section{Existence of Traveling Wave Solution}

In this section, we establish the existence of traveling wave connecting $E_{1}$ and $E_{3}$ in Eq. 2. Assume that Eq. 2 has a solution in the form of $(h(x-c t), q(x-c t))$ such that $(h(-\infty), q(-\infty))=E_{3}$ and $(h(+\infty), q(+\infty))=E_{1}$, where $c$ is the wave speed. Denote $x-c t$ by $\xi$ and substitute $(u(t, x), w(t, x))=(h(\xi), q(\xi))$ into Eq. 2. Thus, Eq. 2 has a pair of traveling wave solutions if and only if it has the following wave equations:

$$
\left\{\begin{array}{l}
h^{\prime \prime}+c h^{\prime}+h p_{1}(h, q)=0 \\
\delta q^{\prime \prime}+c q^{\prime}+q p_{2}(h, q)=0
\end{array}\right.
$$

where $\quad h^{\prime}=\frac{d h}{d \xi} \quad, \quad h^{\prime \prime}=\frac{d^{2} h}{d \xi^{2}} \quad, \quad q^{\prime}=\frac{d q}{d \xi} \quad, \quad q^{\prime \prime}=\frac{d^{2} q}{d \xi^{2}} \quad, \quad p_{1}(h, q)=1-h-\frac{\alpha q}{a+h^{2}}$, $p_{2}(h, q)=s\left(1-\frac{q}{b+h}\right)$.

Assume that $S=[0,1] \times[0, b+1]$ and $S_{0}=\left\{(u, w) \in S, p_{1}(u, w)<0, p_{2}(u, w)>0, w \leq w_{*}\right\}$.

Consider the differential equation

$$
d \frac{d^{2} \varphi}{d \xi^{2}}+c \frac{d \varphi}{d \xi}+m \varphi=0
$$

Suppose that 


$$
c^{2}>4 d m
$$

with $d=\max (1, \delta)$ and $m=\max _{(u, w) \in S}\left(p_{1}(u, w), p_{2}(u, w)\right)$.

Lemma 1. ([9]) Assume that $\varphi: R_{+} \rightarrow R$ is uniformly continuous and Riemann integrable, then $\lim _{\xi \rightarrow \infty} \varphi(\xi)=0$.

Lemma 2. ([10]] Assume that $d, c$ and $m$ are positive constants such that $c^{2}>4 d m$, then for any $\xi>\xi_{0}, \varphi(\xi) \neq 0$ provided that $\varphi$ is a solution of Eq. 4 and $\varphi^{\prime}\left(\xi_{0}\right)=0$.

Lemma 3. Suppose that $(h(\xi), q(\xi))$ is a solution of Eq. 3 satisfying $(h(0), p(0)) \in S_{0}$ and $\left(h^{\prime}(0), q^{\prime}(0)\right)=(0,0)$. If Eq. 5 holds, it follows that

(a) For any $\xi>0, h^{\prime}>0, q^{\prime}<0, u_{*}<h<1,0<q<w_{*}$.

(b) $\lim _{\xi \rightarrow+\infty} h(\xi)=1, \lim _{\xi \rightarrow+\infty} q(\xi)=0$.

(c) $h^{\prime}(\xi)$ and $q^{\prime}(\xi)$ are uniformly bounded.

Proof. It is clear that $h(\xi) \geq 0, q(\xi) \geq 0$ for any $\xi>0$. Note that $h^{\prime \prime}=-h p_{1}(h, q)>0$ and $q^{\prime \prime}=-\frac{q}{\delta} p_{2}(h, q)>0$ at $\xi=0$. Thus, there exists an enough small neighborhood of $\xi=0$ such that $h^{\prime \prime}(\xi)>0, q^{\prime \prime}(\xi)>0$, and $h^{\prime}(\xi)>0, q^{\prime}(\xi)>0$. Suppose that there exists $\bar{\xi}>0$ such that $h^{\prime}(\xi)>0$ on $[0, \bar{\xi})$ and $h^{\prime}(\bar{\xi})=0$. Let $\bar{\xi}$ denote the first such point and $\varphi(\xi)=\lambda_{2} e^{\lambda_{1} \xi}-\lambda_{1} e^{\lambda_{2} \xi}$ be the solution of

$$
\varphi^{\prime \prime}+c \varphi^{\prime}+m \varphi=0
$$

Then, there exist $\varphi(0)=\lambda_{2}-\lambda_{1}, \varphi^{\prime}(0)=0$, where $\lambda_{2}$ and $\lambda_{1}$ are the roots of (6) and condition Eq. 5 is satisfied. Define $\rho(\xi)=e^{c \xi}$. By multiplying Eq. 6 by $\rho h$ and subtracting the product of the first equation of Eq. 3 with $\rho \varphi$, it has

$$
\frac{d\left(\rho\left(h \varphi^{\prime}-h^{\prime} \varphi\right)\right)}{d \xi}+\left(m-p_{1}(h, q)\right)=0 \text {. }
$$

Integrating Eq. 7 from 0 to $\bar{\xi}$, there exist $\rho(\bar{\xi}) h(\bar{\xi}) \varphi^{\prime}(\bar{\xi})+\int_{0}^{\vec{\xi}}\left(m-p_{1}(h, q)\right) \rho(\xi) h(\xi) d \xi=0$ and $\left(m-p_{1}(h, q)\right)>0$.

It leads to a contradiction since $\rho(\bar{\xi})>0, h(\bar{\xi})>0$ and $\varphi(\bar{\xi})>0$. Thus, $h^{\prime}(\xi)>0$ for any $\xi>0$. By applying the same method, it is clear that $q^{\prime}(\xi)<0$. Furthermore, it follows that $u_{*} \leq h(\xi) \leq 1$ and $0 \leq q(\xi) \leq w_{*}$ if $u_{*} \leq h(0) \leq 1$ and $0 \leq q(0) \leq w_{*}$. As a result, there exist $\hat{h}$ and $\hat{q}$ such that $\lim _{\xi \rightarrow+\infty} h(\xi)=\hat{h}, \lim _{\xi \rightarrow+\infty} q(\xi)=\hat{q}$. Meanwhile, from Eq. 3, it has $h^{\prime \prime}+c h^{\prime} \leq \frac{\alpha h q}{h^{2}+a}, \delta q^{\prime \prime}+c q^{\prime} \leq \frac{s q^{2}}{h+b}$. Since $h(\xi)$ and $q(\xi)$ are uniformly bounded, it is clear that there exist two positive constants $\hat{H}, \hat{Q}$ such that

$$
h^{\prime \prime}+c h^{\prime} \leq \hat{H}, \delta q^{\prime \prime}+c q^{\prime} \leq \hat{Q} .
$$


From Eq.8, we obtain the uniform bound of $h^{\prime}(\xi)$ and $q^{\prime}(\xi)$. Consequently, $p^{\prime \prime}(\xi)$ and $q^{\prime \prime}(\xi)$ are also uniformly bounded. Applying Lemma Eq. 3 to $\left(h^{\prime}(\xi), q^{\prime}(\xi)\right)$ and $\left(h^{\prime \prime}(\xi), q^{\prime \prime}(\xi)\right)$, it follows that $\lim _{\xi \rightarrow+\infty} h^{\prime}(\xi)=0, \lim _{\xi \rightarrow+\infty} h^{\prime \prime}(\xi)=0$ and $\lim _{\xi \rightarrow+\infty} q^{\prime}(\xi)=0$, $\lim _{\xi \rightarrow+\infty} q^{\prime \prime}(\xi)=0$. Taking into account $\xi \rightarrow+\infty$ in Eq. 3, it has $f(\hat{H}, \hat{Q})=0$, $g(\hat{H}, \hat{Q})=0$, as a sequence of which it leads to $\hat{H}=1, \hat{Q}=0$. The above results show that any solution which starts at $\xi=0$ in $S_{0}$ approaches to the boundary equilibrium $E_{1}$ as $t \rightarrow+\infty$.

Lemma 4. ([10]) Assume that there exist $\tau_{n, 1}>0, \tau_{n, 2}>0$ such that $\left(h_{n}\left(\tau_{n, 1}\right), q_{n}\left(\tau_{n, 2}\right)\right)=\left(\frac{u_{*}}{2}, \frac{w_{*}}{2}\right)$, then $\lim _{n \rightarrow+\infty} \tau_{n, i}=+\infty, i=1,2$.

Theorem 1. Assume that Eq. 6 is satisfied, then there exists a solution $(h(\xi), q(\xi))$ of Eq. 3 defined in $R$ such that $(h(-\infty), q(-\infty))=E_{*},(h(+\infty), q(+\infty))=E_{1}$ and $u_{*}<h<1,0<q<w_{*}, h^{\prime}>0, q^{\prime}<0$.

Proof. For any $n \in \mathrm{N}$, let $\left(h_{n}(\xi), q_{n}(\xi)\right)$ be the solution of Eq. 3 with the initial conditions, $\quad\left(h_{n}(0), q_{n}(0)\right)=\left(c_{n, 1}, c_{n, 2}\right) \in S_{0} \quad$ and $\quad\left(h_{n}^{\prime}(0), q_{n}^{\prime}(0)\right)=(0,0) \quad$, where $\left(c_{n, 1}, c_{n, 2}\right) \rightarrow\left(u_{*}, w_{*}\right)$ as $n \rightarrow+\infty$. From Lemma 2.3 , for any $n \in \mathrm{N}$ and $\xi>0$, it has $\frac{d h_{n}}{d \xi}(\xi)>0, \frac{d q_{n}}{d \xi}(\xi)<0$ and $\lim _{\xi \rightarrow+\infty} h_{n}(\xi)=1, \lim _{\xi \rightarrow+\infty} q_{n}(\xi)=0$. Thus, it needs to make further step of the traveling wave by considering a sequence of functions which converges into $E_{3}$. For any $n \in \mathrm{N}$ and $\xi \geq \tau_{n, i}, i=1,2$, define $\left(\phi_{n, 1}(\xi), \phi_{n, 2}(\xi)\right)=\left(h_{n}\left(\xi+\tau_{n, 1}\right), q_{n}\left(\xi+\tau_{n, 2}\right)\right)$ for $\xi \geq-\tau_{n, i}, i=1,2$. Since $\left(h_{n}^{\prime}(\xi), q_{n}^{\prime}(\xi)\right)$ is uniformly bounded, then $\left(\phi_{n, 1}^{\prime}(0), \phi_{n, 2}^{\prime}(0)\right)$ is bounded. Thus there is a sequence $\left(\phi_{n_{k}, 1}(\xi), \phi_{n_{k}, 2}(\xi)\right)$ such that $\left(\phi_{n_{k}, 1}^{\prime}(0), \phi_{n_{k}, 2}^{\prime}(0)\right)=\left(h_{n}^{\prime}\left(\tau_{n_{k}, 1}\right), q_{n}^{\prime}\left(\tau_{n_{k}, 2}\right)\right) \rightarrow\left(\theta_{1}, \theta_{2}\right) \quad$ as $k \rightarrow+\infty$ with $\theta_{1} \geq 0$ and $\theta_{2} \leq 0$. Assume that $v=\left(v_{1}, v_{2}\right)$ is the solution of

$$
\left\{\begin{array}{l}
v_{1}^{\prime \prime}+c v_{1}^{\prime}+v_{1} p_{1}\left(v_{1}, v_{2}\right)=0 \\
\delta v_{2}^{\prime \prime}+c v_{2}^{\prime}+v_{2} p_{2}\left(v_{1}, v_{2}\right)=0
\end{array}\right.
$$

satisfying $v(0)=\left(\frac{u_{*}}{2}, \frac{w_{*}}{2}\right)$ and $v^{\prime}(0)=\left(\theta_{1}, \theta_{2}\right)$.Then $\left\{\phi_{n_{k}}\right\}$ converges uniformly into $v(\xi)$ on any bounded neighborhood in $R^{2}$. Thus, $v(\xi) \in S_{0}, v_{1}^{\prime}(\xi) \geq 0$ and $v_{2}^{\prime}(\xi) \leq 0$. For any $\xi \in R$, there in fact exist $v_{1}^{\prime}(\xi)>0$ and $v_{2}^{\prime}(\xi)<0$. Similarly, it is clear that $\lim _{\xi \rightarrow+\infty} v(\xi)=E_{1}$ and $\lim _{\xi \rightarrow+\infty} v^{\prime}(\xi)=(0,0)$. From the uniform convergence of $\left\{\phi_{n_{k}}\right\}$ to $v(\xi)$, it has $\lim _{\xi \rightarrow-\infty} v(\xi)=(\breve{H}, \breve{Q})$, where $\frac{u_{*}}{2} \leq \breve{H} \leq u_{*}, \frac{w_{*}}{2} \leq \breve{Q} \leq w_{*}$. Applying the above theorem, it has $\lim _{\xi \rightarrow-\infty} v^{\prime}(\xi)=(0,0), \lim _{\xi \rightarrow-\infty} v^{\prime \prime}(\xi)=(0,0)$. From Eq. 9, it follows that $p_{1}(\breve{H}, \breve{Q})=0, p_{2}(\breve{H}, \breve{Q})=0$. Thus, $(\breve{H}, \breve{Q})=E_{*}$, that is $\lim _{\xi \rightarrow-\infty} v(\xi)=E_{*}$. The above results show that solution $v(\xi)$ goes to the interior equilibrium $E_{*}$ as $t \rightarrow-\infty$. This completes the proof. 


\section{Numerical Simulations}

In this part, numerical simulations are performed to establish the existence of traveling wave solutions of Eq. 2. All the chosen parameters are $\alpha=0.7, s=0.5, a=9.5$, $b=3$ and $\delta=8$. Then Eq. 2 possesses the equilibrium points: $E_{0}=(0,0), E_{1}=(1,0)$, $E_{2}=(0,3), E_{3}=(0.7394,3.7394)$. By Theorem 1 , there exists a traveling wave solution, which connects $E_{1}$ with $E_{3}$. Using the numerical simulation, the traveling wave solutions of Eq. 2 are shown in Fig. 1 and Fig. 2. It should be stressed that the trajectory of traveling wave solutions moves from $E_{1}$ to $E_{3}$. This confirms the feasibility and correctness of the theoretical analyses.
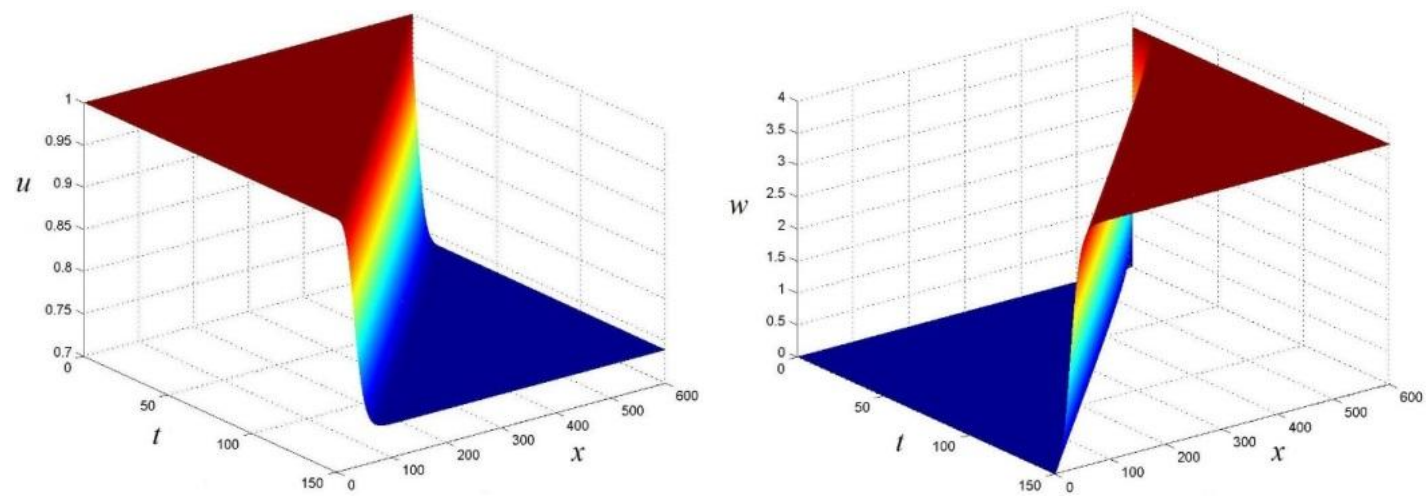

Figure 1. Traveling wave solutions found by numerical integration of Eq.2.
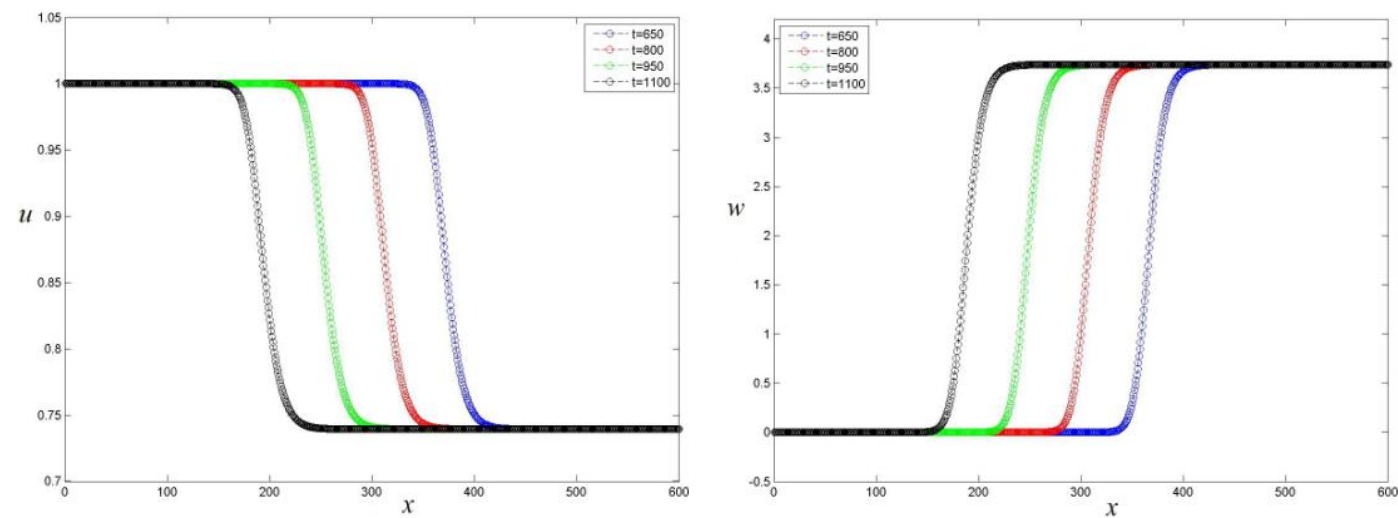

Figure 2. Profiles of traveling wave solutions.

\section{Conclusions}

In this paper, our studied focus of the modified Leslie-Gower model with spatial diffusion and Holling IV schemes is its traveling wave solutions. First, we prove the existence of traveling wave connecting $E_{1}$ and $E_{3}$ in Eq. 2 . On the basis of the above theoretical analyses, the numerical simulations have been to show the correctness of analytic results.

\section{Acknowledgments}

This work was supported by the Scientific Research Fund of Sichuan Provincial Education Department (No. 15ZB0333). 


\section{References}

[1] P.H. Leslie, Some further notes on the use of matrices in population mathematics, Biometrika, 35(1948) 213-245.

[2] P.H. Leslie and J.C. Gower, The properties of a stochastic model for the predator-prey type of interaction between two species, Biometrika, 47(1960) 219-234.

[3] R. Pal, D. Basub and M. Banerjeec, Modeling of phytoplankton allelopathy with Monod-Haldane-type functional response-A mathematical study, BioSystems, 95(2009)243-253, 2009.

[4] B. Dubey, N. Kumari and R.K. Upadhyay, Spatiotemporal pattern formation in a diffusive predator-prey system: an analytical approach, J. Appl.Math. Comput, 31(2009)413-432.

[5] Y.Z. Wang and M. Zhao, Dynamic analysis of an impulsively controlled predator-prey model with Holling type IV functional response, Discrete Dynamics in Nature and Society, 49(2012)213-222.

[6] W.Z. Huang, Problem on minimum wave speed for a Lotka-Volterra reaction-diffusion competition model, J.Dyn.Diffe.Equ, 22(2010)285-297.

[7] C.A. Klausmeier, Regular and irregular patterns in semiarid vegetation, Science, 284(1999)1826-1828.

[8] M. Rietkerk, S.C.Dekker, P. C. de Ruiter, J. van de Koppel, Self-organized patchiness and catastrophic shifts in ecosystems, Science, 305(2004)1962-1929.

[9] L. Hartmut, P.R. Eugene, Asymptotic behaviour of nonlinear systems, The American Mathematical Monthly, 111(2004)864-889.

[10] S. Ahmad, A.C. Lazer and A. Tineo, Traveling waves for a system of equations, Nonlinear Anal-Theor, 68(2008)909-3912. 